\title{
Elevated International Normalized Ratio: A Risk Factor for Portal Vein Thrombosis in Cirrhotic Patients
}

\author{
Eric Omar Then a, d, Vijay Shailendra Are ${ }^{b}$, Michell Lopez-Luciano, ${ }^{\mathrm{a}}$ Rani Bijjamª, \\ Andrew Ofosu ${ }^{\mathrm{c}}$, Andrea Culliford ${ }^{\mathrm{a}}$, Vinaya Gaduputi ${ }^{\mathrm{a}}$
}

\begin{abstract}
Background: Portal vein thrombosis (PVT) is a complication that is commonly seen in patients with cirrhosis and an entity that leads to increased mortality in patients who undergo liver transplantation. This study aims to establish a link between an elevated international normalized ratio (INR) and the presence of PVT in a cohort of cirrhotic patients.

Methods: We retrospectively reviewed the electronic medical records of all patients diagnosed with cirrhosis in SBH Health System from 2013 to 2018. Among these patients we extracted baseline demographic data, laboratory results, co-morbidities and the presence of PVT.

Results: In total there were 268 patients who met our inclusion criteria. Twenty-two patients had PVT, while 246 patients did not. Of the 22 patients with PVT there was a statistically significant increase in INR when compared to patients without PVT. There was also a statistically significant increase in total bilirubin, alkaline phosphatase and platelet count.

Conclusions: Elevated INR levels are associated with the presence of PVT in patients with cirrhosis. These findings suggest a hypercoagulable state and could assist clinicians in risk-stratifying patients when making the decision to initiate anti-coagulation therapy.
\end{abstract}

Keywords: Portal vein thrombosis; Cirrhosis; Anticoagulation; International normalized ratio

\section{Introduction}

The international normalized ratio (INR) is a measure that

Manuscript submitted March 27, 2019, accepted April 17, 2019

aDivision of Gastroenterology and Hepatology, SBH Health System, 4422 Third Ave, Bronx, NY 10457, USA

${ }^{b}$ Department of Internal Medicine, Stormont Vail Health, 1500 SW 10th Ave Topeka, KS 66604, USA

'Division of Gastroenterology and Hepatology, The Brooklyn Hospital Center, Clinical Affiliate of The Mount Sinai Hospital, 121 Dekalb Ave, Brooklyn, NY 11201, USA

${ }^{\mathrm{d} C o r r e s p o n d i n g ~ A u t h o r: ~ E r i c ~ O m a r ~ T h e n, ~ D i v i s i o n ~ o f ~ G a s t r o e n t e r o l o g y ~ a n d ~}$ Hepatology, SBH Health System, 4422 Third Ave, Bronx, NY 10457, USA. Email: ericomarthen27@yahoo.com

doi: https://doi.org/10.14740/gr1179 has long been used to assess coagulation factors in a patient's bloodstream. Specifically, these factors include factors I (fibrinogen), II (prothrombin), V (proaccelerin), VII (proconvertin), and X (Stuart-Prower factor). The aforementioned factors are part of the extrinsic coagulation pathway, which contribute to the process of achieving hemostasis [1]. In practice physicians commonly use this measure to determine a patient's propensity to develop life-threatening bleeding. This is especially true in patients with liver disease, vitamin $\mathrm{K}$ deficiency and those undergoing treatment with warfarin. Protein $\mathrm{C}$ and protein $\mathrm{S}$ are two factors, rather than contributing to coagulation, are the body's natural reservoir of anti-coagulation. These two factors are often overlooked and may be aberrant in conditions that affect INR levels.

Portal vein thrombosis (PVT) is defined as partial or complete obstruction of blood flow that occurs secondary to a thrombus in the portal vein, which results in occlusion of the vessel lumen [2]. With an estimated incidence rate of 0.7 per 100,000 , PVT is a well-established condition found in patients suffering from cirrhosis [3]. Despite this, the etiology of PVT has eluded clinicians for decades. In present days, studies suggest that decreased portal flow velocity, and increased flow volume are considered to be independent risk factors in the development of PVT [4]. No studies however have attempted to establish a relationship between coagulation profile, specifically elevations in INR, and PVT. Given the fact that coagulation factors are produced in the liver, their synthesis is greatly reduced in cirrhotic patients. As mentioned previously, elevations in INR predispose patients to develop life-threatening bleeding. The logic behind this lies in the aberrant production of coagulation factors. There may be a juncture however where protein $\mathrm{C}$ and protein $\mathrm{S}$, the natural anti-coagulants, become more severely affected than the coagulation factors. This in theory will result in a pro-coagulant state, which can predispose cirrhotic patients to develop PVT. This is clinically significant, as early identification of PVT may lead to better outcomes in patient care, decreased length of hospital stay and in turn, reduced mortality.

\section{Methods and Materials}

This is a retrospective single-center observational study. The total period of the study was 5 years, which took place between January 2013 and December 2018. The study was performed 
according to the guidelines dictated by the declaration of Helsinki and was approved by the Institution Review Board (IRB) of St. Barnabas Hospital Health System.

\section{Patient selection}

The data was collected from the electronic medical records of patients and tabulated in Microsoft Excel. Patients diagnosed with cirrhosis (identified by ICD-10 codes) who underwent appropriate imaging (ultrasound abdomen, computed tomography (CT) abdomen with intravenous contrast, or magnetic resonance imaging (MRI) with intravenous contract) were admitted to the hospital floor and intensive care unit. Patients who underwent $\mathrm{CT}$ of the abdomen without intravenous contrast or MRI without intravenous contrast were excluded from the study given their poor sensitivity in detecting PVT. Similarly, patients who had missing information, namely INR levels, were also excluded from the study. The diagnosis of PVT was made in patients who had absent blood flow in the portal vein, cavernous transformation of the portal vein, or the presence of solid, hyperechoic material within the portal vein. Because the treatment of PVT consists of initiating anticoagulation, patients who were taking warfarin or direct oral anticoagulants (DOACs) were excluded from our study. A total of 268 patients were finally included and divided into two study groups.

\section{Evaluation of results}

Our study intended to establish a correlation between the presence of PVT and INR levels in patients with cirrhosis. We also looked at these correlations separately across genders and ethnicities.

\section{Group division}

The study group consisted of patients with the presence of PVT (group 2) and the control group consisted of patients without PVT (group 1). All lab values were measured closest to the date of imaging study performed.

\section{Data collection}

Baseline demographic data including age, gender, and ethnicity were collected for all patients in the study. We collected data on the patients' body mass index (BMI), complete blood count, comprehensive metabolic panel, liver function test, and coagulation profile (including INR, prothrombin time (PT), and partial thromboplastin time (PTT)). We also calculated model for end-stage liver disease (MELD) scores for each patient within both study groups. Child-Pugh scores were not included due to the considerable heterogeneity in documentation of subjective variables of ascites and hepatic encephalopathy. Data regarding social history including usage of tobacco, heroin, and alco- hol were also included in the analysis. In addition we reviewed endoscopy reports evaluating for the presence of esophageal varices in each patient. Finally we included the presence of co-morbidities found in patients from both study groups such as hypertension, diabetes mellitus, hepatitis B, hepatitis $\mathrm{C}$ and human immunodeficiency virus.

\section{Statistical methods}

Results were reported as the means with standard deviation for most variables and $95 \%$ confidence interval for some variables. For comparisons of continuous variables between the two study groups we used the $t$-test. Categorical variables for the two study groups were compared by Chi-square analysis using the Pearson test. A two-tailed $\mathrm{P}$ value of $<0.05$ was considered to be statistically significant.

\section{Results}

In total there were 268 patients who met our inclusion criteria who were admitted to the hospital between January 2013 and December 2018. Of these patients, 22 (8\%) were found to have PVT and 246 (92\%) were found to have patent portal veins. Out of the total study population 180 patients were male, and 88 were female. There were 138 Hispanics, 71 African Americans, 57 Caucasians, one Asian and one person of Indian origin in the study population.

Baseline characteristics for patients with PVT and for those without PVT are tabulated in Table 1. There was no observable statistically significant difference in age, gender or ethnicity between the two groups. Likewise, there was no statistically significant difference in both groups between users of alcohol, tobacco and heroin.

Our results showed that patients with PVT had a statistically significant increased INR level when compared to patients without PVT $(\mathrm{P}$ value $=0.04)$. The mean INR for patients with PVT was 1.45 , whilst the mean for patients with patent portal veins was 1.3.

Similarly, in patients with PVT there was a statistically significant increase in bilirubin $(4.8 \mathrm{mg} / \mathrm{dL}$ vs. $2.6 \mathrm{mg} / \mathrm{dL}$; P value $=0.021)$, alkaline phosphatase $(281 \mathrm{IU} / \mathrm{L}$ vs. $130 \mathrm{IU} / \mathrm{L}$; $\mathrm{P}$ value $=0.0001)$ and platelet count $\left(224 \times 10^{3} / \mathrm{uL}\right.$ vs. $131 \times$ $10^{3} / \mathrm{uL} ; \mathrm{P}$ value $=0.0001$ ) when compared to those without PVT. In addition, patients with PVT had statistically significant lower sodium levels than those without PVT $(135 \mathrm{mEq} / \mathrm{L}$ vs. $137 \mathrm{mEq} / \mathrm{L}$; P value $=0.041)$. While the mean MELD score in patients with PVT was higher than that in those without portal thrombosis, these findings were not statistically significant $(16.5$ vs. $14.1 ; \mathrm{P}$ value $=0.144)($ Table 2$)$.

Out of the 22 patients with confirmed PVT, nine patients had esophageal variceal bleeding confirmed by esophagogastroduodenoscopy (EGD). In the control group, 70 out of 246 patients had esophageal variceal bleeding confirmed by EGD. There was no statistically significant difference in the presence of variceal bleeding between the two study groups $(\mathrm{P}$ value $=$ 0.21) (Table 3). 
Table 1. Patient Characteristics

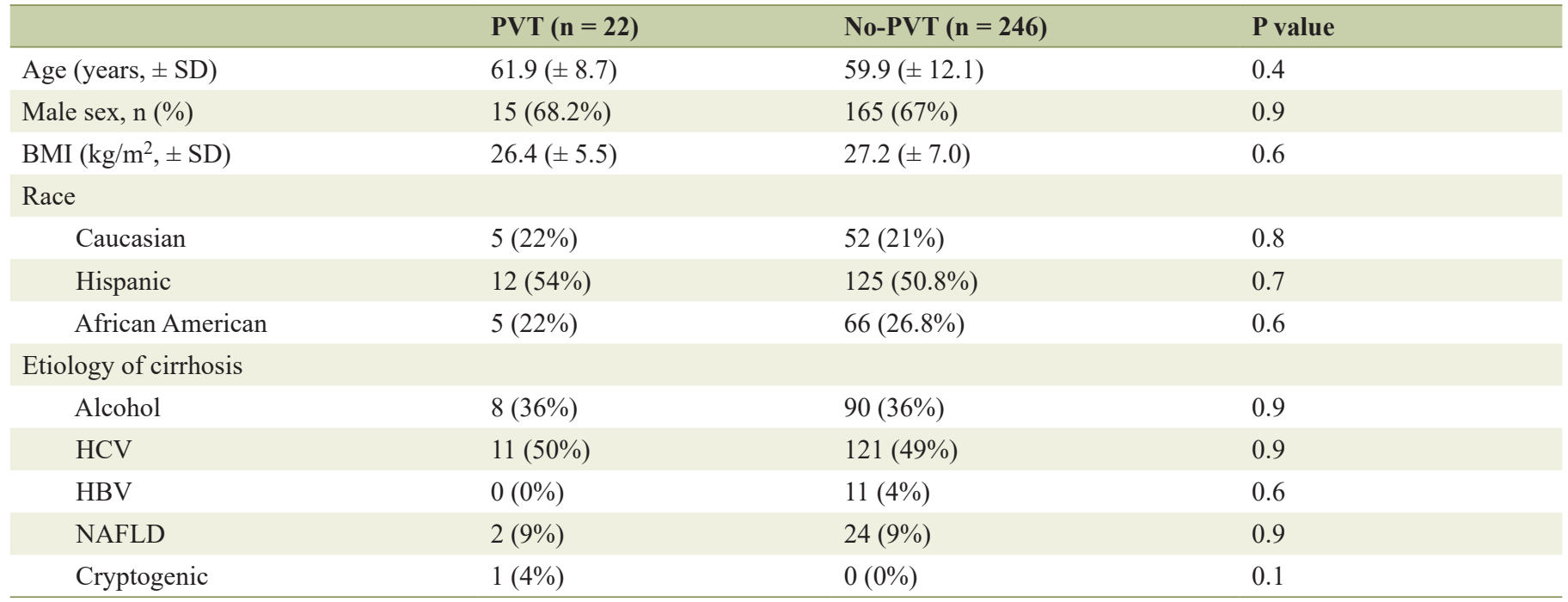

PVT: portal vein thrombosis; BMI: body mass index; HCV: hepatitis C virus; HBV: hepatitis B virus; NAFLD: non-alcoholic fatty liver disease.

Table 2. Serologic Markers

\begin{tabular}{llll} 
& PVT $(\mathbf{n}=\mathbf{2 2})$ & No-PVT $(\mathbf{n}=\mathbf{2 4 6})$ & P value \\
\hline INR $( \pm \mathrm{SD})$ & $1.45( \pm 0.39)$ & $1.3( \pm 0.35)$ & 0.04 \\
Sodium $(\mathrm{mEq} / \mathrm{L}, \pm \mathrm{SD})$ & $135( \pm 5.2)$ & $137( \pm 4.5)$ & 0.04 \\
Platelet $\left(10^{3} / \mathrm{uL}, \pm \mathrm{SD}\right)$ & $224( \pm 196)$ & $131( \pm 94)$ & 0.0001 \\
Albumin $(\mathrm{g} / \mathrm{dL}, \pm \mathrm{SD})$ & $2.5( \pm 0.93)$ & $2.7( \pm 0.78)$ & 0.15 \\
Creatinine $(\mathrm{mg} / \mathrm{dL}, \pm \mathrm{SD})$ & $1.1( \pm 1.0)$ & $1.3( \pm 1.5)$ & 0.5 \\
ALP $(\mathrm{IU} / \mathrm{L}, \pm \mathrm{SD})$ & $281( \pm 376)$ & $130( \pm 92)$ & 0.0001 \\
Total bilirubin $(\mathrm{mg} / \mathrm{dL}, \pm \mathrm{SD})$ & $2.6( \pm 4.08)$ & 0.02 \\
MELD $( \pm \mathrm{SD})$ & $16.5( \pm 7.8)$ & $14.1( \pm 7.3)$ & 0.1 \\
\hline
\end{tabular}

PVT: portal vein thrombosis; INR: international normalized ratio; ALP: alkaline phosphatase; MELD: model for end-stage liver disease.

Hepatitis $\mathrm{C}$ was the etiology responsible for cirrhosis in the majority of patients in both groups (50\% in study group, and $49 \%$ in control group). This was followed by alcohol $(36 \%$ in study group, and $28 \%$ in control group), and non-alcoholic fatty liver disease (9\% in study group, and $13 \%$ in control group). There was no statistically significant association between any of these etiologies with the presence of PVT.

Table 3. Co-Morbid Conditions

\begin{tabular}{llll}
\hline & PVT $(\mathbf{n}=22)$ & No-PVT (n= 246) & P value \\
\hline Hypertension (\%) & $13(59 \%)$ & $137(55 \%)$ & 0.7 \\
Diabetes (\%) & $8(36 \%)$ & $85(34 \%)$ & 0.8 \\
Heroin abuse (\%) & $11(50 \%)$ & $88(35 \%)$ & 0.1 \\
Tobacco use (\%) & $14(63 \%)$ & $156(63 \%)$ & 0.9 \\
HIV (\%) & $2(9 \%)$ & $40(16 \%)$ & 0.5 \\
EV (\%) & $9(40 \%)$ & $70(28 \%)$ & 0.2 \\
\hline
\end{tabular}

PVT: portal vein thrombosis; HIV: human immunodeficiency virus; EV: esophageal varices.

\section{Discussion}

Conventionally liver disease is associated with coagulopathy and bleeding tendencies. In recent years more and more evidence is pointing towards the often-ignored thrombophilic, hypercoagulable tendencies that accompany liver pathology. Worsening liver function leads to decreased synthesis of various proteins including numerous anti-coagulants such as protein $\mathrm{C}$, protein $\mathrm{S}$, and anti-thrombin 3. Dwindling anticoagulant proteins in the blood stream may counterbalance the bleeding tendencies caused by the loss of pro-coagulants $[5,6]$. In compensated cirrhosis there is a delicate and vulnerable balance between coagulopathic and thrombophilic tendencies. Various precipitating factors can tip this balance one way or the other leading to distinct complications. Advancing liver disease is associated with a higher tendency for hypercoagulability. In a prospective study, Zocco et al reported that reduction in levels of anticoagulant proteins was strongly related to severity of liver cirrhosis according to the MELD scoring system [7]. In their study, D-dimer was significantly elevated in patients with a MELD score greater 
than or equal to 13 . In addition, there was a negative correlation between levels of $\mathrm{D}$-dimer and both anti-thrombin and protein $\mathrm{C}$ levels. PVT is one of the most common clinical manifestations of hypercoagulability in liver disease.

Epidemiologically, PVT has an estimated prevalence of $0.6-15.8 \%$ of all patients with cirrhosis or portal hypertension [8]. The etiology of cirrhosis seems to play a role in the development of cirrhosis. In one cohort of 45 cirrhotic patients, PVT was found to have a higher incidence rate in patients with hepatitis B virus [9]. In our cohort of 22 patients with PVT, over $50 \%$ of patients had hepatitis $\mathrm{C}$ virus. These findings as mentioned previously were not statistically significant however. Regarding ethnicity, one large cohort of 3,642 patients found that African Americans and Hispanics had a statistically significant lower prevalence of PVT when compared to Caucasian patients at initial presentation. At time of transplantation, African Americans also had a decreased prevalence, when compared to Caucasians. Interestingly, Hispanic patients in this subgroup were found to have a higher prevalence of PVT when compared to African Americans and Caucasians [10]. In our cohort the majority of patients (54\%) were Hispanic. These findings were also not statistically significant and may likely be attributed to the large demographic of Hispanic patients that our facility serves.

In present days, there are multiple classification systems to determine the different types of PVT. The Baveno VI classification proposed in 2015 is perhaps the most widely used and stratifies PVT by location, etiology of underlying liver disease, degree of occlusion, and onset (acute vs. chronic). Type 1 refers to thrombosis of the portal vein trunk, type 2 refers to thrombosis of one branch $(2 \mathrm{a})$ or both branches $(2 \mathrm{~b})$, and type 3 refers to thrombosis of both the portal vein trunk and branches [11]. One shortcoming of this classification system is the omission of functional consequences of PVT. Sarin et al recently proposed a new classification system that adds functional features of PVT to the previous parameters delineated in the Baveno VI classification [12].These functional features are defined as symptomatic PVT and asymptomatic PVT.

Thrombus formation depends on three factors as explained by Virchow's triad: stasis, hypercoagulability and endothelial injury.

Portal flow velocity less than $15 \mathrm{~cm} / \mathrm{s}$ is an established risk factor for the development of PVT. Decreased portal vein flow might actually be a manifestation of advancing liver cirrhosis with progressive fibrosis and alteration in the microcirculation. Portal flow velocity is inversely related to Child-Pugh score. Flow velocity is lower in patients in Child-Pugh class $\mathrm{C}$ compared to patients in class B or A [13].

More than two-thirds of cirrhosis patients with PVT have some evidence of inherited thrombophilia [14]. Prothrombin (PT) gene 20210A mutation is associated with a more than five-fold increased risk of developing PVT [15]. PT gene mutation by itself is considered a weak risk factor for venous thrombosis [16] but in the presence of other risk factors can pose a considerable risk for thrombus formation [17]. Factor $\mathrm{V}$ Leiden mutation and methylenetetrahydrofolate reductase C667T mutation are more frequently detected in cirrhotic patients with PVT compared to those without PVT [18].

Markers of endothelial dysfunction like von Willebrand factor, P-selectin and isoprostanes are elevated in patients with cirrhosis. Endothelial cell dysfunction may contribute to thrombus formation in cirrhotic patients; further studies are needed to prove causality.

PVT is considered to be a negative prognostic factor in advanced liver disease. Extensive PVT after liver transplantation has been shown to be positively associated with higher mortality $[19,20]$. One study in particular found that patients undergoing liver transplantation with PVT had longer operation time, increased need for transfusions and lower survival rate than those without PVT [21]. Another study echoed these findings showing that patients with PVT had a higher posttransplant mortality than those without PVT. Interestingly there was not an associated increase in mortality among patients who were on the transplant waiting list [22].

PVT can be a consequence or cause of decompensation in cirrhosis. It is asymptomatic in $50 \%$ of patients with cirrhosis and is symptomatic in the other half. If symptomatic it can lead to the following complications.

Thrombosis of the mesenteric vein as a result of PVT can lead to intestinal ischemia and infarction. PVT is one of the differentials for acute abdominal pain in a cirrhotic patient and abdominal CT must be considered in the diagnostic workup [23]. If left untreated mesenteric vein thrombosis can result in severe gastrointestinal bleeding, and in some cases the development of peritonitis, which carries with it a high rate of mortality [24].

The presence of PVT is oftentimes associated with portal hypertension. This will result in port-systemic shunting due to the increased pressure that develops secondary to the obstructed vessel. In turn, this will cause the development of esophageal and gastric varices, upper gastrointestinal bleeding and subsequent mortality [25]. Further, one study found that patients with cirrhosis and PVT had a $10 \%$ higher rate of bleeding when compared to cirrhotic patients without PVT [26]. In our study, patients with PVT had a higher likelihood of developing esophageal variceal bleeding than those without PVT, but these findings were not statistically significant.

Septic thrombophlebitis of the portal vein, also known as pylephlebitis, is a complication of PVT that occurs in conjunction with an intra-abdominal infection. It is characterized by suppurative thrombosis of the portal vein, fever, abdominal pain and bacteremia [27]. Infections associated with its development include diverticulitis, pancreatitis and appendicitis. Regarding treatment, most cases of pylephlebitis can be managed conservatively with antibiotics and anticoagulation. In cases that result in bowel ischemia, surgical resection may be employed [28].

Portal cholangiopathy refers to biliary changes occurring secondary to extrahepatic PVT. The decreased blood flow associated with PVT causes the genesis of venous collateral pathways that cause biliary obstruction and deformity, namely changes in the paracholedocal, epicholedocal, and cholecystic veins. Clinically this will result in biliary-type abdominal pain, jaundice, and elevated levels of bilirubin and alkaline phosphatase [29].

Anticoagulation for PVT in cirrhosis is a management dilemma especially in non-transplant patients. As per the Baveno IV consensus, anticoagulation should be considered in potential liver transplantation candidates with thrombosis of the 
main portal trunk or those with progressive PVT. The ultimate goal of initiating treatment is to facilitate liver transplantation and reduce post-transplant morbidity and mortality [30]. As for non-transplant cirrhotic patients, aggressive therapy should be considered in those with underlying inflammatory conditions and in those at high risk to develop mesenteric ischemia [14]. There is no consensus, however, on treatment for PVT in non-cirrhotic patients. The role of anticoagulation in PVT is based on findings from five studies [18, 31-34] with a total patient population of 176 . In these studies, with treatment $8-50 \%$ patients showed complete thrombus resolution, and partial resolution was observed in $33-45 \%$. Thrombus progression was seen in less than $10 \%$ of the patients. In all these studies low-molecular-weight heparin (LMWH) and vitamin $\mathrm{K}$ antagonists were used for treatment. DOACs are becoming a more popular alternative considering there is no requirement for monitoring. Data from two studies $[35,36]$ report that incidence of bleeding episodes with use of DOAC in cirrhosis is not higher compared to traditional anticoagulation. The major concern with anticoagulation in cirrhosis is the risk for causing a new bleeding episode and increasing the severity of one if it were to happen. Identifying risk factors involved in PVT pathophysiology might help identify high risk subgroups who will benefit the most from anticoagulation on risk-benefit ratio.

Our study was hindered by multiple limitations. The first limitation was the retrospective nature of our study, which carries with it inherent weaknesses. Another weakness occurred in terms of the power of our study. Despite going back 5 years, we were only able to identify 22 patients with PVT. Also in our study we diagnosed cirrhosis by imaging studies and not through liver biopsy, which is the gold standard. Future, prospective studies are required to validate our findings. In addition, other retrospective studies correlating the presence of PVT and levels of other markers suggestive of thrombophilia such as protein $\mathrm{C}$, protein $\mathrm{S}$ or factor $\mathrm{V}$ Leiden, would also be helpful in supporting our hypothesis.

In conclusion, our study found that elevated INR levels were associated with the presence of PVT in cirrhotic patients. It is important to identify patients that may be afflicted by PVT given the varied set of complications that are invariably associated with it. This is especially true in patients who are possible liver transplant recipients, and who have higher rates of mortality in the post-transplant period. Regarding management, the initiation of anti-coagulation has long been a point of contention due to the higher risk of bleeding that is associated with cirrhotic patients. Stratifying these patients by INR levels may assist clinicians in making the decision to treat or withhold anticoagulation therapy on a case-by-case basis.

\section{Acknowledgments}

None.

\section{Financial Disclosure}

None.

\section{Conflict of Interest}

None of the authors have any financial conflict of interest.

\section{Informed Consent}

Not applicable.

\section{Author Contributions}

EOT, VSA, MLL, AO, and RB are involved in conception and design; EOT and VG are involved in interpretation of the data; EOT, VSA, MLL, RB and AO are involved in drafting of the article; $\mathrm{VG}$ and $\mathrm{AC}$ are involved in revision of the article for important intellectual content; $\mathrm{VG}$ and $\mathrm{AC}$ are involved in final approval of the article.

\section{References}

1. Mucino-Bermejo J, Carrillo-Esper R, Uribe M, MendezSanchez N. Coagulation abnormalities in the cirrhotic patient. Ann Hepatol. 2013;12(5):713-724.

2. Ponziani FR, Zocco MA, Campanale C, Rinninella E, Tortora A, Di Maurizio L, Bombardieri G, et al. Portal vein thrombosis: insight into physiopathology, diagnosis, and treatment. World J Gastroenterol. 2010;16(2):143155.

3. Rajani R, Bjornsson E, Bergquist A, Danielsson A, Gustavsson A, Grip O, Melin T, et al. The epidemiology and clinical features of portal vein thrombosis: a multicentre study. Aliment Pharmacol Ther. 2010;32(9):1154-1162.

4. Qi X, Han G, Fan D. Management of portal vein thrombosis in liver cirrhosis. Nat Rev Gastroenterol Hepatol. 2014;11(7):435-446.

5. Tripodi A, Salerno F, Chantarangkul V, Clerici M, Cazzaniga M, Primignani M, Mannuccio Mannucci P. Evidence of normal thrombin generation in cirrhosis despite abnormal conventional coagulation tests. Hepatology. 2005;41(3):553-558.

6. Bosch J, Reverter JC. The coagulopathy of cirrhosis: myth or reality? Hepatology. 2005;41(3):434-435.

7. Zocco MA, Di Stasio E, De Cristofaro R, Novi M, Ainora ME, Ponziani F, Riccardi L, et al. Thrombotic risk factors in patients with liver cirrhosis: correlation with MELD scoring system and portal vein thrombosis development. J Hepatol. 2009;51(4):682-689.

8. Mantaka A, Augoustaki A, Kouroumalis EA, Samonakis DN. Portal vein thrombosis in cirrhosis: diagnosis, natural history, and therapeutic challenges. Ann Gastroenterol. 2018;31(3):315-329.

9. Cagin YF, Atayan Y, Erdogan MA, Dagtekin F, Colak C. Incidence and clinical presentation of portal vein thrombosis in cirrhotic patients. Hepatobiliary Pancreat Dis Int. 2016;15(5):499-503.

10. Bezinover D, Reeder E, Aziz F, Saner F, McQuillan P, 
Kadry Z, Riley T, et al. African Americans have a lower prevalence of portal vein thrombosis at the time of liver transplantation. HPB (Oxford). 2017;19(7):620-628.

11. de Franchis R, Baveno VIF. Expanding consensus in portal hypertension: Report of the Baveno VI Consensus Workshop: Stratifying risk and individualizing care for portal hypertension. J Hepatol. 2015;63(3):743-752.

12. Sarin SK, Philips CA, Kamath PS, Choudhury A, Maruyama H, Nery FG, Valla DC. Toward a Comprehensive New Classification of Portal Vein Thrombosis in Patients With Cirrhosis. Gastroenterology. 2016;151(4):574-577 e573.

13. Zironi G, Gaiani S, Fenyves D, Rigamonti A, Bolondi L, Barbara L. Value of measurement of mean portal flow velocity by Doppler flowmetry in the diagnosis of portal hypertension. J Hepatol. 1992;16(3):298-303.

14. von Kockritz L, De Gottardi A, Trebicka J, Praktiknjo M. Portal vein thrombosis in patients with cirrhosis. Gastroenterol Rep (Oxf). 2017;5(2):148-156.

15. Valla DC, Cazals-Hatem D. Vascular liver diseases on the clinical side: definitions and diagnosis, new concepts. Virchows Arch. 2018;473(1):3-13.

16. Poort SR, Rosendaal FR, Reitsma PH, Bertina RM. A common genetic variation in the 3'-untranslated region of the prothrombin gene is associated with elevated plasma prothrombin levels and an increase in venous thrombosis. Blood. 1996;88(10):3698-3703.

17. Margaglione M, Di Minno G, Grandone E, Vecchione G, Celentano E, Cappucci G, Giordano M, et al. Raised plasma fibrinogen concentrations in subjects attending a metabolic ward - relation to family history and vascular risk factors. Thromb Haemost. 1995;73(4):579-583.

18. Amitrano L, Brancaccio V, Guardascione MA, Margaglione M, Iannaccone L, D'Andrea G, Marmo R, et al. Inherited coagulation disorders in cirrhotic patients with portal vein thrombosis. Hepatology. 2000;31(2):345-348.

19. Janssen HL, Wijnhoud A, Haagsma EB, van Uum SH, van Nieuwkerk CM, Adang RP, Chamuleau RA, et al. Extrahepatic portal vein thrombosis: aetiology and determinants of survival. Gut. 2001;49(5):720-724.

20. Gayowski TJ, Marino IR, Doyle HR, Echeverri L, Mieles $\mathrm{L}$, Todo S, Wagener M, et al. A high incidence of native portal vein thrombosis in veterans undergoing liver transplantation. J Surg Res. 1996;60(2):333-338.

21. Lendoire J, Raffin G, Cejas N, Duek F, Barros Schelotto P, Trigo P, Quarin C, et al. Liver transplantation in adult patients with portal vein thrombosis: risk factors, management and outcome. HPB (Oxford). 2007;9(5):352356.

22. Englesbe MJ, Schaubel DE, Cai S, Guidinger MK, Merion RM. Portal vein thrombosis and liver transplant survival benefit. Liver Transpl. 2010;16(8):999-1005.
23. Amitrano L, Guardascione MA, Brancaccio V, Margaglione M, Manguso F, Iannaccone L, Grandone E, et al. Risk factors and clinical presentation of portal vein thrombosis in patients with liver cirrhosis. J Hepatol. 2004;40(5):736741.

24. Kumar S, Sarr MG, Kamath PS. Mesenteric venous thrombosis. N Engl J Med. 2001;345(23):1683-1688.

25. Trebicka J, Strassburg CP. Etiology and complications of portal vein thrombosis. Viszeralmedizin. 2014;30(6):375380 .

26. Tsochatzis EA, Senzolo M, Germani G, Gatt A, Burroughs AK. Systematic review: portal vein thrombosis in cirrhosis. Aliment Pharmacol Ther. 2010;31(3):366-374.

27. Garrett A, Carnish E, Das N, Slome M, Measley R. Once universally fatal: pylephlebitis. Am J Med. 2014;127(7):595-597.

28. Choudhry AJ, Baghdadi YM, Amr MA, Alzghari MJ, Jenkins DH, Zielinski MD. Pylephlebitis: a Review of 95 Cases. J Gastrointest Surg. 2016;20(3):656-661.

29. Khuroo MS, Rather AA, Khuroo NS, Khuroo MS. Portal biliopathy. World J Gastroenterol. 2016;22(35):79737982.

30. Lazzaroni M. R. De Franchis, Portal Hypertension IVproceedings from the 4th Baveno International Consensus Workshop, Blackwell Publishing, Malden, MA, USA (2006), 424 pp., Price US\$ 125.00, ISBN: 1-4051-39404. Dig Liver Dis. 2006;38(12):942-943.

31. Korula J, Yellin A, Kanel GC, Nichols P. Portal vein thrombosis complicating endoscopic variceal sclerotherapy. Convincing further evidence. Dig Dis Sci. 1991;36(8):1164-1167.

32. Saeed ZA, Stiegmann GV, Ramirez FC, Reveille RM, Goff JS, Hepps KS, Cole RA. Endoscopic variceal ligation is superior to combined ligation and sclerotherapy for esophageal varices: a multicenter prospective randomized trial. Hepatology. 1997;25(1):71-74.

33. Amitrano L, Brancaccio V, Guardascione MA, Margaglione M, Sacco M, Martino R, De Nucci C, et al. Portal vein thrombosis after variceal endoscopic sclerotherapy in cirrhotic patients: role of genetic thrombophilia. Endoscopy. 2002;34(7):535-538.

34. Francoz C, Belghiti J, Vilgrain V, Sommacale D, Paradis $\mathrm{V}$, Condat B, Denninger MH, et al. Splanchnic vein thrombosis in candidates for liver transplantation: usefulness of screening and anticoagulation. Gut. 2005;54(5):691-697.

35. Hoolwerf EW, Kraaijpoel N, Buller HR, van Es N. Direct oral anticoagulants in patients with liver cirrhosis: A systematic review. Thromb Res. 2018;170:102-108.

36. Intagliata NM, Maitland $\mathrm{H}$, Pellitier S, Caldwell SH. Reversal of direct oral anticoagulants for liver transplantation in cirrhosis: A step forward. Liver Transpl. 2017;23(3):396-397. 